\title{
BMJ Open Provision of care for chronic kidney disease by non-nephrologists in a developing nation: a national survey
}

\author{
S Al Shamsi, ${ }^{1}$ A Al Dhanhani, ${ }^{1}$ M M Sheek-Hussein, ${ }^{2}$ O Bakoush ${ }^{1}$
}

To cite: Al Shamsi S,

Al Dhanhani A,

Sheek-Hussein MM, et al. Provision of care for chronic kidney disease by nonnephrologists in a developing nation: a national survey. BMJ Open 2016;6:e010832. doi:10.1136/bmjopen-2015010832

- Prepublication history and additional material is available. To view please visit the journal (http://dx.doi.org/ 10.1136/bmjopen-2015010832).

Received 16 December 2015 Revised 8 May 2016 Accepted 8 June 2016

\section{CrossMark}

${ }^{1}$ Department of Internal Medicine, College of Medicine \& Health Sciences, United Arab Emirates University, Al Ain, United Arab Emirates

${ }^{2}$ Institute of Public Health, College of Medicine \& Health Sciences, United Arab Emirates University, Al Ain, United Arab Emirates

Correspondence to Dr 0 Bakoush;

Omran.Bakoush@med.lu.se

\section{ABSTRACT}

Objectives: The prevalence of chronic kidney disease (CKD) in developing countries has increased dramatically. This study aimed to explore the practice patterns of non-dialysis-dependent CKD care in an affluent developing country.

Settings: Primary and specialised healthcare facilities of public and private sectors in the United Arab Emirates.

Participants: 159 non-nephrologist physicians practising in the United Arab Emirates.

Interventions: A 28-item online self-administered questionnaire based on CKD clinical practice guidelines.

Primary and secondary outcome measures: The physicians' approach to identifying and managing patients with CKD.

Results: The survey was completed by 159 nonnephrologists, of whom 135 reported having treated patients with CKD. Almost all the respondents screen patients with hypertension and diabetes for CKD, but one-third of them do not screen patients with cardiovascular disease and elderly patients for CKD. The use of accurate CKD screening tests (estimated glomerular filtration rate and albumin/creatinine ratio) was suboptimal $(77 \%$ and $59 \%$ of physicians used the procedures, respectively). One-third of the physicians do not offer treatment with inhibitors of the reninangiotensin system to patients with CKD, and only $66 \%$ offer antilipid treatment. In general, the primary healthcare physicians are more familiar than secondary healthcare physicians with the diagnosis and management of patients with CKD.

Conclusions: We identified substantial physiciandeclared deficiencies in the practice of identifying and managing early CKD. Integration of quality CKD care within the healthcare system is required to face the increasing burden of CKD in the United Arab Emirates and possibly in other developing nations.

\section{INTRODUCTION}

Chronic kidney disease (CKD) is a global public health problem. ${ }^{1}$ In developing countries, including the United Arab Emirates (UAE), CKD is widespread because of the increasing prevalence of diabetes,

\section{Strengths and limitations of this study}

- This national multicentre cross-sectional study included non-nephrologist physicians from the three major healthcare providers: Abu Dhabi Health Services Company, Dubai Health Authority and Ministry of Health, United Arab Emirates.

- The questionnaire items were based on the internationally widely accepted chronic kidney disease clinical practice guidelines for the Evaluation and Management of Chronic Kidney Disease (KDIGO).

- The small size of the study and the possibility that some physicians' responses might have been based on their knowledge rather than on their actual practice may limit the generalisability of the findings.

hypertension and coronary artery disease..$^{2-6}$ In the UAE, the age-adjusted prevalence of diabetes is $19 \%$ of the population and an additional $12.5 \%$ of the population has impaired glucose tolerance. ${ }^{7}$ In alignment with the epidemic of metabolic and cardiovascular diseases (CVDs), the prevalence of CKD in UAE is estimated to be very high. ${ }^{6-10}$

Early CKD is relatively asymptomatic but is nevertheless associated with serious comorbidities and increased cardiovascular mortality. ${ }^{11}$ So it is important to provide quality care for early CKD in order to prevent death and avoid the need for dialysis and kidney transplantation. ${ }^{12} 13$ Owing to the growing number of patients with CKD and the limited number of nephrologists, physicians in primary and specialised healthcare (SHC) have to play an important role in providing this quality care to patients with CKD. ${ }^{12}$

Providing quality CKD care includes regular screening of high-risk individuals, such as patients with diabetes, hypertension or CVD, ${ }^{13}{ }^{14}$ using the more accurate CKD diagnostic tools, such as estimated glomerular filtration rate (eGFR) and the urine albumin/creatinine ratio (ACR). Quality 
CKD care also requires implementation of effective preventive strategies, such as drugs that block the renin -angiotensin system, which can delay or prevent CKD complications. $^{13} 14$

Despite two decades of widely accepted CKD clinical practice guidelines, such as the Kidney Disease Outcomes Quality Initiative (KDOQI) and continuing medical education for physicians, recent reports from many affluent developed countries indicate that CKD care remains suboptimal. ${ }^{15} 16$ The deficiencies in CKD care are expected to be more serious in developing nations that lack sufficient funds for quality CKD care, ${ }^{17} 18$ and there is little or no information about the CKD care in affluent developing countries with wellfunded healthcare systems, such as the UAE.

For many years, the UAE healthcare authorities have had a modern healthcare service that is covered by a compulsory health insurance scheme for the entire population. ${ }^{19}$ Access to pharmacy services and modern diagnostic laboratory support are also available at the point of care. ${ }^{20}{ }^{21}$ Nevertheless, awareness of patients and physicians of CKD is essential for effective CKD clinical practice. ${ }^{22}$

The aim of this study was to explore how nonnephrologist physicians screen, diagnose and manage CKD in the UAE, which has seen a dramatic rise in the prevalence of CKD. ${ }^{8}$

\section{METHODS}

This is a cross-sectional study based on an electronic survey of physicians. A 28-item self-administered questionnaire was designed to determine the physicians' approach to identification (8 questions) and management (11 questions) of patients with CKD (see online supplementary file). Nine other questions were used to identify the demographics and characteristics of the study participants. The themes of the questions were based on KDIGO recommendations and guidelines. ${ }^{13}$ The questionnaire was reviewed by three nephrologists and four internists at the College of Medicine, UAE University (UAEU) to assess the face validity of the survey items and to provide feedback on the clarity and conciseness of the questionnaire. We then posted the questionnaire using an online survey programme that allows respondents to answer the questions but does not allow duplication of responses. The link to the survey, along with a cover letter, was emailed to a test group of primary healthcare $(\mathrm{PHC})$ physicians $(\mathrm{n}=4)$ and attending physicians of internal medicine $(\mathrm{n}=5)$, Al Ain, UAE. No problems were identified in the pilot study and therefore no changes were made to the questionnaire. The link to the online survey and the cover letter were then sent by electronic mail to the Dubai branch of the Emirates Medical Association (EMA), and to the medical directors within Abu Dhabi health services (SEHA) institutes for forwarding to their member physicians. The directors could decide whether to forward the survey, and physicians' participation was voluntary.
Responses were anonymous and no identifying data were collected. Three reminders were sent on our behalf by the EMA Office and the medical directors of Abu Dhabi health services. As an incentive, participants were offered a workshop to discuss the results of the questionnaire and CKD management. Responses were collected from 26 September 2013 to 5 March 2014. The survey's first page contained information on the rationale of the study and statements ensuring the confidentiality of respondents. The participants were given the choice to decline or to proceed to answer the survey questions. The survey questions allow for no response as an option for every question, and the participants can stop participation at any time. Completion of the survey signified informed consent.

\section{Statistical methods}

We summarised the questionnaire responses using descriptive statistics. The proportions were calculated based on the total number of respondents for each question. The $\chi^{2}$ or Fisher's exact test was used to assess the independence of categorical variables, as appropriate. We employed logistic regression analysis to examine the variations in the practice of CKD care by locality and the provider characteristics as independent variables. The dependent variables were the screening for CKD in patients with hypertension, diabetes mellitus or CVD and in elderly patients, the use of eGFR and ACR in the diagnosis of CKD, and the initiation of treatment with ACE inhibitors. All variables with a value of $p<0.1$ in the univariate analysis were entered into a stepwise multiple regression analysis. Analyses were performed using SPSS V.18.0 (IBM, Chicago, Illinois, USA) and p values $<0.05$ were considered statistically significant.

\section{RESULTS}

\section{Participant characteristics}

We do not know the number of physicians who received the questionnaire, so we could not compute the exact response rate. Based on the number of physicians with active email accounts registered on the website of the Dubai branch of EMA and those practising at participating Abu Dhabi health services institutes, up to 536 physicians could have received the email invitation for participation in the study. We received responses from 185 physicians $(40.5 \%$ female), giving a response rate of at least 34\%. We excluded 26 questionnaires: 3 incomplete questionnaires, 6 that were from non-physicians (2 nurses, 3 dentists and 1 non-clinician), 13 that were from nephrologists and 4 from physicians who had limited or no contact with patients (3 radiologists and 1 pathologist). Of the remaining 159 respondents, the 135 physicians who declared that they see patients with CKD in their practice were used in the analysis (figure 1).

Of the 135 respondents, $72(41.5 \%)$ were from PHC facilities and the others were from SHC institutions, including all medical subspecialties that encounter 
kidney patients except nephrologists. The geographical distribution of respondents is shown in figure 2. Dubai and the Northern Emirates were classified as locality A (60.7\% of respondents), whereas Abu Dhabi and $\mathrm{Al}$ Ain areas were labelled as locality B (39.3\% of respondents). The overall median age of the physicians was 45 years (25-60), of whom $66 \%$ of them had been practising for more than 5 years in the UAE (table 1 ).

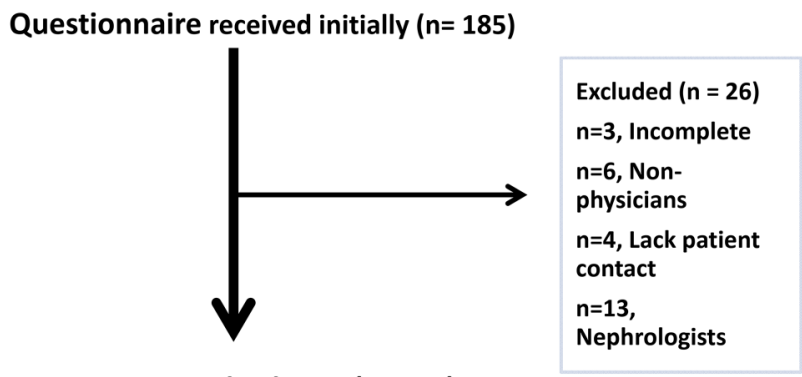

Non-nephrologist $(n=159)$

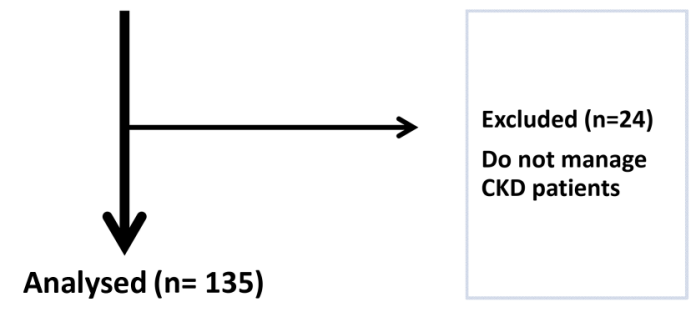

Figure 1 Flow diagram of exclusions and inclusions. CKD, chronic kidney disease.
Sixty-three per cent $(n=85)$ reported using CKD guidelines and $82 \%(n=109)$ had access to nephrology consultation. CKD guidelines were being used more frequently by PHC physicians $(75 \% ; \mathrm{n}=40)$ than by SHC physicians $(56 \% ; \mathrm{n}=45 ; \mathrm{p}=0.031)$. Over the past year, $41 \%$ of the physicians $(n=55)$ had been seeing five or more patients with CKD per week (table 1).

\section{Screening and diagnosis}

Table 2 shows the CKD care patterns among PHC and SHC physicians. Almost all respondents stated that they screen patients with hypertension or diabetes for CKD (94\% and $96 \%$ of physicians, respectively), but fewer screen for CKD in patients with CVD $(76 \%, \mathrm{p}<0.001)$ and elderly patients $(68 \%, \mathrm{p}<0.001$; table 2$)$. Seventyseven per cent of respondents use eGFR as a screening tool for CKD and $19.3 \%$ use serum creatinine alone. Fifty-nine per cent use ACR for measurement of proteinuria, $11 \%$ use 24-hour urine collection and $7 \%$ use urine dipstick. However, only $49.6 \%$ use both eGFR and ACR in the diagnosis of CKD, while $38 \%$ of the respondents rely on nephrology consultations to confirm the diagnosis of CKD.

\section{Referral}

An eGFR $<60 \mathrm{~mL} / \mathrm{min} / 1.73 / \mathrm{m}^{2}$ or a serum creatinine $>150 \mu \mathrm{mol} / \mathrm{L}$ prompts $55 \%$ of the respondents to refer

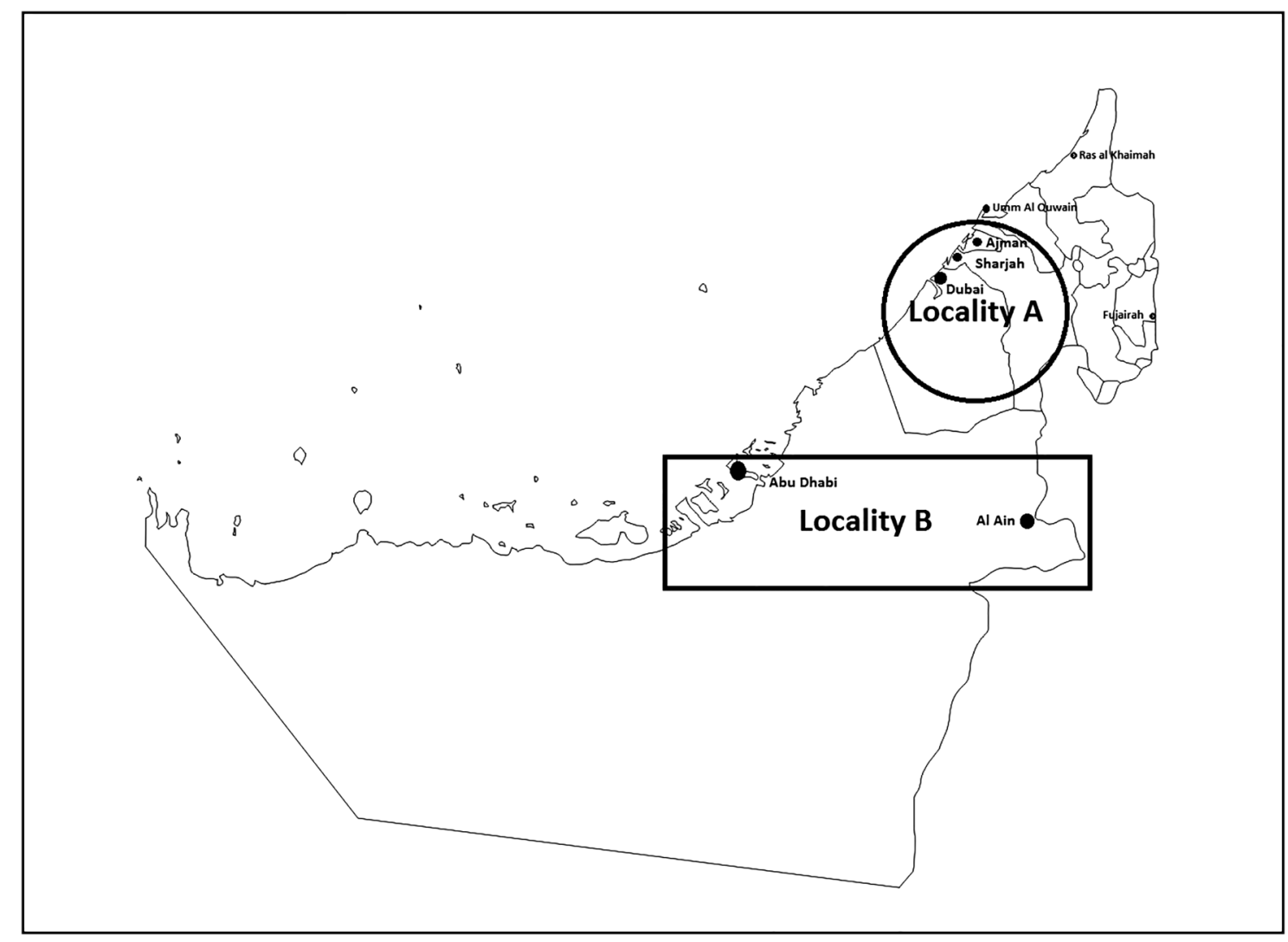

Figure 2 The respondents' practice sites in the UAE. UAE, United Arab Emirates. 
their patients to nephrology. Fifty-nine per cent consider significant proteinuria an indication for referral while $25 \%$ refer if there is associated haematuria.

Table 1 The characteristics of the practice of 135 non-nephrologist physicians who see patients with CKD at $\mathrm{PHC}$ or SHC facilities in the United Arab Emirates

\begin{tabular}{lllll}
\hline & Total & \multicolumn{3}{l}{ PHC vs SHC facilities } \\
\cline { 2 - 5 } & $\begin{array}{l}\text { Per } \\
\text { cent (n) }\end{array}$ & $\begin{array}{l}\text { PHC } \\
\text { (\%) }\end{array}$ & $\begin{array}{l}\text { SHC } \\
\text { (\%) }\end{array}$ & $\begin{array}{l}\text { p } \\
\text { Value }\end{array}$ \\
\hline Affiliation of & 100 & 40.0 & 60.0 & \\
physician & $(135)$ & & & \\
Age (years) & 45 & 45 & 45 & 0.74 \\
Sex (M/F) & $76 / 55$ & $23 / 30$ & $53 / 25$ & 0.005 \\
Locality (A/B) & $82 / 53$ & $29 / 25$ & $53 / 28$ & 0.21 \\
Service >5 years & 66.4 & 70.4 & 63.8 & 0.46 \\
$\begin{array}{l}\text { See >5 patients with } \\
\text { CKD/week }\end{array}$ & 40.7 & 35.2 & 44.4 & 0.29 \\
$\begin{array}{l}\text { Use CKD guidelines } \\
\text { Access to }\end{array}$ & 63.4 & 75.5 & 55.6 & 0.031 \\
nephrology services & 82.0 & 75.9 & 86.1 & 0.14 \\
\hline CKD, chronic kidney disease; F, female; M, male; PHC, primary \\
healthcare; SHC, secondary healthcare.
\end{tabular}

\section{Management}

Ninety-six per cent of the respondents consider CKD a risk factor for CVD, but only $66 \%$ of them provide antilipid treatment, $67 \%$ prescribe weight reduction and $80 \%$ recommend smoking cessation to their patients. Eighty-five per cent consider a blood pressure control $\leq 130 / 80$ as an optimal target, $13 \%$ use a blood pressure target of $>130 / 80$ and $1.5 \%$ have no specific blood pressure target. Seventy-six per cent initiate ACE inhibitors or angiotensin receptor blockers $(n=103)$, but in practice $61 \%$ of those who offer ACE inhibitors estimated that at least half of their patients were on these medications.

\section{Association of CKD care with physician and practice characteristics}

Univariate logistic regression analysis is shown in table 3. The PHC physicians were more familiar than SHC physicians with the use of eGFR in the diagnosis of CKD $(\mathrm{OR}=2.8 ; 95 \%$ CI 1.1 to $7.1 ; \mathrm{p}=0.028)$. However, physicians working in locality $\mathrm{B}$ were four times more likely to use eGFR than those in locality A $(\mathrm{OR}=4.5 ; 95 \%$ CI 1.6 to $12.5 ; \mathrm{p}=0.005)$. Multivariate regression analysis

Table 2 The pattern of chronic kidney disease diagnosis and management of 135 non-nephrologist physicians at PHC and $\mathrm{SHC}$ facilities in the United Arab Emirates

\begin{tabular}{|c|c|c|c|c|}
\hline & \multirow{2}{*}{$\begin{array}{l}\text { Total } \\
\text { Per cent }\end{array}$} & \multicolumn{3}{|c|}{ PHC vs SHC facilities } \\
\hline & & PHC (\%) & SHC (\%) & p Value \\
\hline \multicolumn{5}{|l|}{ Screening } \\
\hline HTN & 94.1 & 98.1 & 91.4 & 0.10 \\
\hline DM & 96.3 & 98.1 & 95.1 & 0.35 \\
\hline CVD & 75.6 & 79.6 & 72.8 & 0.37 \\
\hline Elderly (>60 years) & 68.1 & 75.9 & 63.0 & 0.11 \\
\hline \multicolumn{5}{|l|}{ Diagnostic tools } \\
\hline \multicolumn{5}{|l|}{ For level of kidney function } \\
\hline Serum creatinine alone & 19.3 & 11.1 & 24.7 & 0.051 \\
\hline eGFR & 77.0 & 87.0 & 70.4 & 0.024 \\
\hline \multicolumn{5}{|l|}{ For proteinuria } \\
\hline ACR & 59.3 & 63.0 & 56.8 & 0.47 \\
\hline 24-hour urine collection & 11.1 & 09.3 & 12.1 & 0.57 \\
\hline Urine dipstick alone & 06.7 & 03.7 & 08.6 & 0.26 \\
\hline \multicolumn{5}{|l|}{ Referral to nephrology } \\
\hline \multicolumn{5}{|l|}{ Level of kidney function } \\
\hline eGFR<60 & 43.9 & 43.4 & 44.3 & 0.80 \\
\hline eGFR $<45$ & 14.4 & 18.9 & 11.4 & 0.26 \\
\hline eGFR<30 & 22.7 & 26.4 & 20.3 & 0.45 \\
\hline $\mathrm{Cr}>150$ & 12.1 & 05.7 & 16.5 & 0.055 \\
\hline $\mathrm{Cr}>300$ & 01.5 & 01.9 & 01.3 & 0.70 \\
\hline \multicolumn{5}{|l|}{ Proteinuria } \\
\hline With or without haematuria & 70.2 & 61.7 & 76.1 & 0.10 \\
\hline Only with haematuria & 29.8 & 38.3 & 23.9 & 0.10 \\
\hline \multicolumn{5}{|l|}{ Treatment } \\
\hline Offer antilipid & 65.9 & 70.4 & 63.0 & 0.37 \\
\hline Weight reduction & 67.4 & 79.6 & 59.3 & 0.013 \\
\hline Smoking cessation & 80.7 & 85.2 & 77.8 & 0.28 \\
\hline BP goal <130/80 & 85.5 & 96.3 & 77.9 & 0.003 \\
\hline ACEl initiation & 76.3 & 87.0 & 69.1 & 0.017 \\
\hline
\end{tabular}


Table 3 Univariate logistic regression analysis of the quality of CKD care in relation to respondents' characteristics

$$
\text { OR } \quad 95 \% \mathrm{Cl} \quad \text { p Value }
$$

Screening for CKD in patients with hypertension, diabetes or CVD and those aged $>60$ years

\begin{tabular}{llll} 
Age & 1.07 & 0.53 to 2.17 & 0.86 \\
Female gender & 1.09 & 0.54 to 2.21 & 0.81 \\
Locality B & 1.47 & 0.72 to 2.99 & 0.29 \\
PHC practice & 2.02 & 0.98 to 4.16 & 0.06 \\
Duration of practice & 1.42 & 0.69 to 2.92 & 0.35 \\
Use CKD guidelines & 1.18 & 0.58 to 2.39 & 0.65 \\
Access to nephrology & 0.83 & 0.34 to 2.02 & 0.68 \\
Use of eGFR & & & \\
Age & 0.80 & 0.35 to 1.82 & 0.59 \\
Female gender & 1.72 & 0.73 to 4.02 & 0.21 \\
Locality B & 4.46 & 1.59 to 12.51 & 0.005 \\
PHC practice & 2.83 & 1.12 to 7.14 & 0.028 \\
Duration of practice & 0.76 & 0.317 to 1.83 & 0.54 \\
Use CKD guidelines & 1.85 & 0.82 to 4.17 & 0.14 \\
Access to nephrology & 1.19 & 0.41 to 3.50 & 0.75 \\
Use of ACR & & & \\
Age & 1.39 & 0.68 to 2.84 & 0.37 \\
Female gender & 2.01 & 0.97 to 4.17 & 0.06 \\
Locality B & 1.08 & 0.53 to 2.18 & 0.83 \\
PHC practice & 1.29 & 0.64 to 2.62 & 0.48 \\
Duration of practice & 1.23 & 0.60 to 2.55 & 0.57 \\
Use CKD guidelines & 1.41 & 0.70 to 2.87 & 0.34 \\
Access to nephrology & 1.22 & 0.49 to 3.02 & 0.67 \\
Initiation of ACEI & & & \\
Age & 0.72 & 0.32 to 1.63 & 0.43 \\
Female gender & 1.83 & 0.76 to 4.39 & 0.18 \\
Locality B & 2.33 & 0.96 to 5.67 & 0.06 \\
PHC practice & 3.00 & 1.19 to 7.55 & 0.02 \\
Duration of practice & 1.60 & 0.70 to 3.66 & 0.26 \\
Use CKD guidelines & 2.03 & 0.907 to 4.54 & 0.08 \\
Access to nephrology & 1.73 & 0.54 to 5.49 & 0.35 \\
Global CKD Care Score & & & \\
Age & 0.95 & 0.47 to 1.91 & 0.88 \\
Female gender & 1.60 & 0.79 to 3.21 & 0.19 \\
Locality B & 2.15 & 1.06 to 4.35 & 0.03 \\
PHC practice & 2.29 & 1.13 to 4.62 & 0.02 \\
Duration of practice & 1.34 & 0.65 to 2.75 & 0.43 \\
Use CKD guidelines & 2.31 & 1.12 to 4.74 & 0.02 \\
Access to nephrology & 0.89 & 0.37 to 2.17 & 0.80 \\
\hline ACE ACE nhbs & &
\end{tabular}

ACEI, ACE inhibitors; ACR, albumin/creatinine ratio; CKD, chronic kidney disease; CVD, cardiovascular disease; eGFR, estimated glomerular filtration rate; locality B refers to Abu Dhabi and Al Ain areas; $\mathrm{PHC}$, primary healthcare.

confirmed that both the locality $(\mathrm{OR}=4.18 ; \mathrm{p}=0.007$, table 4) and PHC practice $(\mathrm{OR}=2.59 ; \mathrm{p}=0.05$, table 4$)$ are independently associated with the use of eGFR. In general, the use of ACR in the diagnosis of CKD was infrequent in both healthcare localities $(59 \%, \mathrm{OR}=1.08$; $\mathrm{p}=0.83$, not significant).

PHC physicians were more familiar with the initiation of therapy with ACE inhibitors than SHC physicians $(\mathrm{OR}=3.0 ; \mathrm{p}=0.02)$. Familiarity was also associated with the use of CKD clinical guidelines $(\mathrm{OR}=2.0 ; \mathrm{p}=0.08)$, but multivariate regression analysis shows a strong association with PHC practice $(\mathrm{OR}=2.58 ; \mathrm{p}=0.02$, table 4$)$ but not with the use of CKD clinical guidelines.

We included eight dichotomous (done or not done) items with face validity to examine the practices of diagnosis and management of CKD (figure 3 ). We found that PHC and SHC physicians differ in their use of eGFR and ACE inhibitors (figure 3). An overall CKD care score was computed by adding the eight items: only 32 physicians $(23.7 \%$ ) obtained a score of $8 / 8$. The multivariate regression analysis shows that the PHC physicians, irrespective of the healthcare locality and the use of CKD guidelines, had better performance than SHC physicians $(\mathrm{OR}=2.29 ; 95 \%$ CI 1.13 to $4.62 ; \mathrm{p}=0.02$, table 4).

\section{DISCUSSION}

Our findings indicate that provision of CKD care by non-nephrologists to non-dialysis-dependent patients is suboptimal in the UAE but comparable to some developed countries. Despite the high prevalence of CKD in the region, ${ }^{2} 913$ a substantial proportion of responding physicians do not screen for CKD in elderly patients and in patients with CVDs, who are at high risk for CKD. This lack of appreciation of CKD risk factors by physicians and their dependence on less accurate methods, such as serum creatinine alone or urine dipstick for diagnosis of CKD, could contribute to delay in the diagnoses of CKD and to suboptimal CKD care. ${ }^{23-25}$ The eGFR and urinary ACR are recommended because of their greater sensitivity and precision in detecting early stages of CKD, and their more effective prediction of progression to kidney failure or cardiovascular death. ${ }^{13}$ This under usage of ACR and eGFR for diagnosis of CKD could be due to the physicians' unfamiliarity with these diagnostic tools or the absence of local laboratory and clinical protocols for their use in routine clinical practice. $^{26-28}$ The rare use of ACR indicates a notable deficiency in the physicians' familiarity with the diagnostic tools for identification of CKD. ${ }^{1327}$ Referral to nephrologist services is usually indicated when eGFR is $<30 \mathrm{~mL} / \mathrm{min} 1.73 / \mathrm{m}^{2} .{ }^{13}$ The preference for early referral reported in this study may indicate that nonnephrologist physicians are uncertain of how to manage patients with CKD. ${ }^{1325}$ This notion is supported by the finding that about one-fourth of physicians do not initiate treatment with ACE inhibitors for patients with CKD.

The perceived suboptimal CKD care in this study is in agreement with recent laboratory evidence of a high frequency of undocumented CKD in the UAE and a delay in nephrology consultations. ${ }^{20}$ Our study findings indicate that PHC facilities in the UAE provide better quality CKD care than non-nephrology SHC clinics (figure 3). The ease of access to nephrology consultation at SHC facilities could contribute to this discrepancy in the practices of CKD care. ${ }^{21}{ }^{29}$ Also, the competing demands of patients with multiple 
Table 4 Multivariate logistic regression analysis of the quality of CKD care in relation to respondents' characteristics

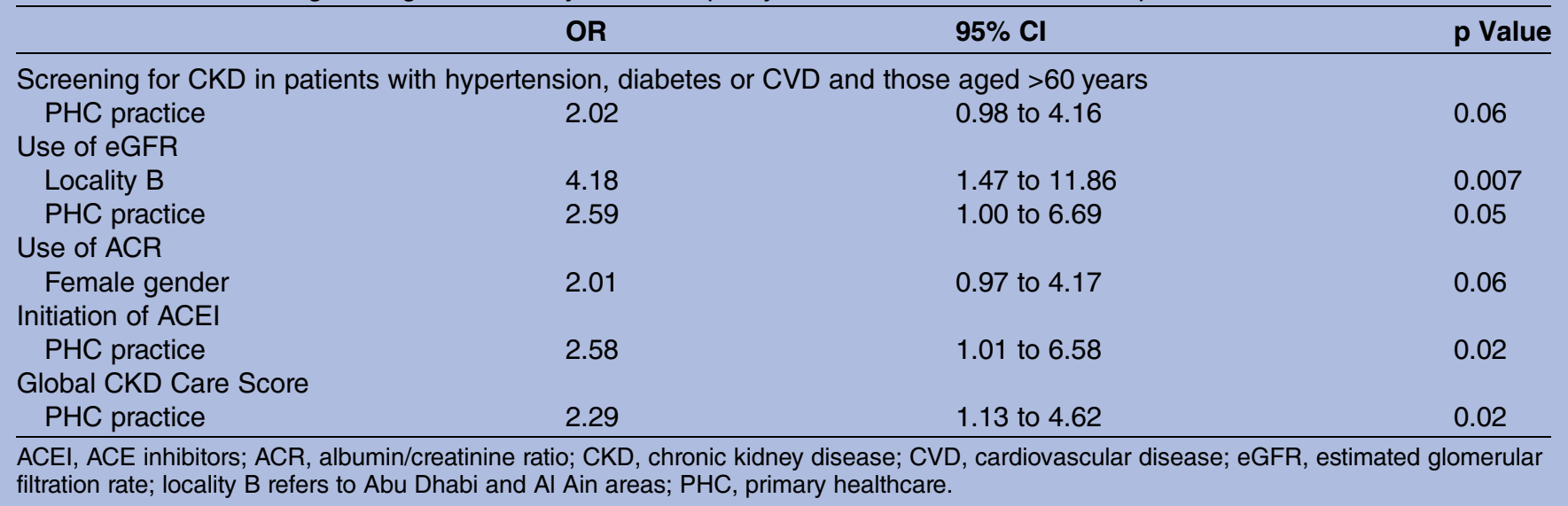

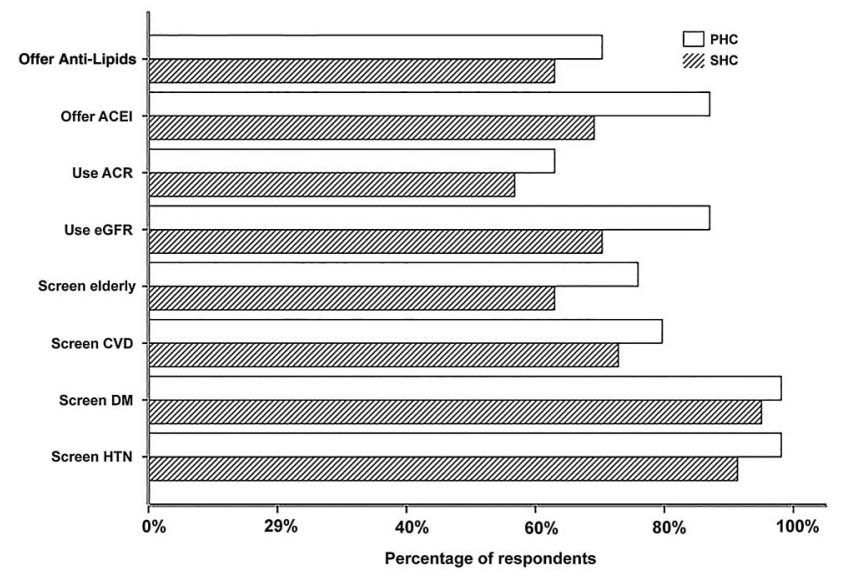

Figure 3 The performance of physicians at PHC and SHC facilities in screening, diagnosis and treatment of CKD. ACEI, ACE inhibitors; ACR, albumin/creatinine ratio; CKD, chronic kidney disease; CVD, cardiovascular disease; DM, diabetes mellitus; eGFR, estimated glomerular filtration rate; HTN, hypertention; $\mathrm{PHC}$, primary healthcare; $\mathrm{SHC}$, specialised healthcare.

comorbidities in SHC would prioritise down CKD care. Other factors related to the healthcare system (eg, insurance cover, practice style and cultural factors) are also potential barriers to optimal delivery of CKD care. $^{22} 29-31$

The approach for optimal CKD care should be multidisciplinary and focus on early identification and management of CKD in patients at high risk. ${ }^{32} 33$ This strategy might need the establishment of specialised CKD clinics and implementation of local protocols for CKD care. ${ }^{34-37}$ Therefore, guidance by healthcare authorities is needed to improve the quality of CKD care in the UAE. A focused national public health programme for CKD care similar to the programmes that have proven their efficacy, such as those for tuberculosis, hypertension and diabetes, should be considered. ${ }^{10}$

Despite vigorous efforts to generate a robust physician response rate, the small size of our sample limits the generalisability of the findings. It is likely that some of the targeted physicians were not monitoring their emails, ${ }^{38}$ but we do not know how much this contributed to the low response rate. It is also possible that physicians with a heavy workload may not have had the time to complete the survey. ${ }^{39}$ A low response rate could result in overestimation or underestimation of CKD awareness in the UAE. However, the per cent distribution of respondents in the relevant specialties working in SHC (60\%) and PHC (40\%) reflects the corresponding percentages in the country, and thus the nonresponse is most likely random. ${ }^{40}$ The other limitation is that while the study focused on describing the patterns of management of patients with CKD, some physicians might have responded based on their knowledge rather than on their actual practice. Moreover, they may not have accurately recalled their practice patterns, or may have attempted to provide what they perceived as the correct answer. Therefore, the study results might have underestimated the deficit in the quality of CKD care. Interviewing physicians and patients with CKD and reviewing medical records might provide additional insight into CKD care in the UAE.

In conclusion, although the study finding indicates deficiencies in the provision of early CKD care, the current state of CKD care remains largely unknown in the UAE, which has a large CKD population. We identified physicians' unawareness of CKD risk factors and unfamiliarity with recommended CKD diagnostic tools and treatment as important shortcomings. Implementation of a specialised primary CKD healthcare programme could overcome these deficiencies.

Acknowledgements The authors wish to thank the directors and secretaries of the Emirates Medical Society, Abu Dhabi ambulatory healthcare, Tawam and Al-Ain hospitals for their assistance in forwarding the web survey to their members. They also acknowledge the writing assistance of Dr Amin Bredan in the preparation of the manuscript.

Contributors SAS, AAD and OB designed the study, analysed the data and drafted the manuscript. MMS-H contributed to the design of the study, analysis of the data and drafting of the manuscript. All authors read and approved the final manuscript. 
Funding This work was supported by grants from CMHS, United Arab Emirates University.

Competing interests None declared.

Ethics approval The Human Ethical Committee at Al Ain district, United Arab Emirates University approved this study (Protocol No. 13/33).

Provenance and peer review Not commissioned; externally peer reviewed.

Data sharing statement No additional data are available.

Open Access This is an Open Access article distributed in accordance with the Creative Commons Attribution Non Commercial (CC BY-NC 4.0) license, which permits others to distribute, remix, adapt, build upon this work noncommercially, and license their derivative works on different terms, provided the original work is properly cited and the use is non-commercial. See: http:// creativecommons.org/licenses/by-nc/4.0/

\section{REFERENCES}

1. Lozano R, Naghavi M, Foreman K, et al. Global and regional mortality from 235 causes of death for 20 age groups in 1990 and 2010: a systematic analysis for the Global Burden of Disease Study 2010. Lancet 2012;380:2095-128.

2. Alhyas L, McKay A, Balasanthiran A, et al. Prevalences of overweight, obesity, hyperglycaemia, hypertension and dyslipidaemia in the Gulf: systematic review. JRSM Short Rep 2011;2:55.

3. WHO. Country cooperation strategy for WHO and United Arab Emirates 2012-2017. Country cooperation strategy. Regional Office for the Eastern Mediterranean. World Health Organization. 2012. http://www.who.int/iris/handle/10665/113226

4. Nugent RA, Fathima SF, Feigl AB, et al. The burden of chronic kidney disease on developing nations: a 21st century challenge in global health. Nephron Clin Pract 2011:118:c269-77.

5. Malik M, Bakir A. Prevalence of overweight and obesity among children in the United Arab Emirates. Obes Rev 2007;8:15-20.

6. Hassanien AA, Al-Shaikh F, Vamos EP, et al. Epidemiology of end-stage renal disease in the countries of the Gulf Cooperation Council: a systematic review. JRSM Short Rep 2012;3:38.

7. Guariguata L. Contribute data to the 6th edition of the IDF Diabetes Atlas. Diabetes Res Clin Pract 2013;100:280-1.

8. Mokdad AH, Jaber S, Aziz Ml, et al. The state of health in the Arab world, 1990-2010: an analysis of the burden of diseases, injuries, and risk factors. Lancet 2014;383:309-20.

9. Farag YM, Kari JA, Singh AK. Chronic kidney disease in the Arab world: a call for action. Nephron Clin Pract 2012;121:c120-3.

10. Hajat C, Harrison O, Shather Z. A profile and approach to chronic disease in Abu Dhabi. Global Health 2012;8:18.

11. Gullion CM, Keith DS, Nichols GA, et al. Impact of comorbidities on mortality in managed care patients with CKD. Am J Kidney Dis 2006;48:212-20.

12. Stevens LA, Li S, Wang C, et al. Prevalence of CKD and comorbid illness in elderly patients in the United States: results from the Kidney Early Evaluation Program (KEEP). Am J Kidney Dis 2010;55 (Suppl 2):S23-33.

13. Improving Global Outcomes (KDIGO) CKD Work Group. KDIGO 2012 clinical practice guideline for the evaluation and management of chronic kidney disease. Kidney Int Suppl 2013;3:1-150.

14. Stevens LA, Schmid CH, Zhang YL, et al. Development and validation of GFR-estimating equations using diabetes, transplant and weight. Nephrol Dial Transplant 2010;25:449-57.

15. Bello AK, Levin A, Manns BJ, et al. Effective CKD care in European countries: challenges and opportunities for health policy. Am J Kidney Dis 2015;65:15-25.

16. Razavian M, Heeley EL, Perkovic V, et al. Cardiovascular risk management in chronic kidney disease in general practice (the AusHEART study). Nephrol Dial Transplant 2012;27:1396-402.

17. Jha V. Current status of chronic kidney disease care in southeast Asia. Semin Nephrol 2009;29:487-96.

18. Garcia-Garcia G, Jha V, Tao Li PK, et al. Chronic kidney disease (CKD) in disadvantaged populations. Clin Kidney J 2015;8:3-6.
19. Badrinath $\mathrm{P}, \mathrm{Al}$-Shboul Q, Zoubeidi T, et al. Measuring the health of the nation: United Arab Emirates health and lifestyle survey 2000. Fac Med Health Sci Coll Econ Al Ain 2002:1-170.

20. Richards N, Hassan M, Saleh AK, et al. Epidemiology and referral patterns of patients with chronic kidney disease in the Emirate of Abu Dhabi. Saudi J Kidney Dis Transpl 2015;26:1028-34.

21. U.S.-U.A.E. Business Council. The U.A.E. Healthcare Sector. Health care Report Update. Washington DC, USA: U.A.E. Business Council, 2014

22. Jurkovitz CT, Elliott D, Li S, et al. Physician utilization, risk-factor control, and CKD progression among participants in the Kidney Early Evaluation Program (KEEP). Am J Kidney Dis 2012;59(Supp 2):S24-33

23. Boulware LE, Troll MU, Jaar BG, et al. Identification and referral of patients with progressive CKD: a national study. Am J Kidney Dis 2006;48:192-204.

24. Minutolo R, De Nicola L, Mazzaglia G, et al. Detection and awareness of moderate to advanced CKD by primary care practitioners: a cross-sectional study from Italy. Am J Kidney Dis 2008:52:444-53.

25. Fox CH, Brooks A, Zayas LE, et al. Primary care physicians' knowledge and practice patterns in the treatment of chronic kidney disease: an Upstate New York Practice-based Research Network (UNYNET) study. J Am Board Fam Med 2006:19:54-61.

26. Abouchacra S, Chaaban A, Gebran N, et al. GFR estimation in the morbidly obese pre- and postbariatric surgery: one size does not fit all. Int Urol Nephrol 2013;45:157-62.

27. Al-Maskari F, El-Sadig M, Obineche E. Prevalence and determinants of microalbuminuria among diabetic patients in the United Arab Emirates. BMC Nephrol 2008;9:1.

28. Taal MW. Chronic kidney disease in general populations and primary care: diagnostic and therapeutic considerations. Curr Opin Nephrol Hypertens 2013;22:593-8.

29. Koornneef EJ, Robben PB, Al Seiari MB, et al. Health system reform in the Emirate of Abu Dhabi, United Arab Emirates. Health Policy 2012;108:115-21.

30. Barakat-Haddad C, Siddiqua A. Primary health care use and health care accessibility among adolescents in the United Arab Emirates. East Mediterr Health J 2015;21:171-84.

31. Hajat C, Harrison O, Al Siksek Z. Weqaya: a population-wide cardiovascular screening program in Abu Dhabi, United Arab Emirates. Am J Public Health 2012;102:909-14.

32. Plantinga LC, Tuot DS, Powe NR. Awareness of chronic kidney disease among patients and providers. Adv Chronic Kidney Dis 2010;17:225-36.

33. Whaley-Connell A, Shlipak MG, Inker LA, et al. Awareness of kidney disease and relationship to end-stage renal disease and mortality. Am J Med 2012;125:661-9.

34. Martínez-Ramírez HR, Cortés-Sanabria L, Rojas-Campos E, et al. Multidisciplinary strategies in the management of early chronic kidney disease. Arch Med Res 2013;44:611-15.

35. Karunaratne $\mathrm{K}$, Stevens $\mathrm{P}$, Irving $\mathrm{J}$, et al. The impact of pay for performance on the control of blood pressure in people with chronic kidney disease stage 3-5. Nephrol Dial Transplant 2013;28:2107-16.

36. Mendu ML, Schneider LI, Aizer AA, et al. Implementation of a CKD checklist for primary care providers. Clin J Am Soc Nephrol 2014;9:1526-35.

37. Baynouna LM, Shamsan Al, Ali TA, et al. A successful chronic care program in Al Ain-United Arab Emirates. BMC Health Serv Res 2010;10:47.

38. Edwards PJ, Roberts I, Clarke MJ, et al. Methods to increase response to postal and electronic questionnaires. Cochrane Database Syst Rev 2009;(3):MR000008.

39. Delva MD, Kirby JR, Knapper CK, et al. Postal survey of approaches to learning among Ontario physicians: implications for continuing medical education. BMJ 2002;325:1218.

40. Holbrook A, Krosnick JA, Pfent A. The causes and consequences of response rates in surveys by the news media and government contractor survey research firms. Adv Telephone Surv Methodol 2007:499-528. 